\title{
A hierarchical scheme for cooperativity and folding in proteins
}

\author{
Alex Hansen, * Mogens H. Jensen, Kim Sneppen and Giovanni Zocchi \\ Niels Bohr Institute and NORDITA, Blegdamsvej 17, DK-2100 Ø, Denmark
}

(October 26, 2018)

\begin{abstract}
We propose a protein model based on a hierarchy of constraints that force the protein to follow certain pathways when changing conformation. The model exhibits a first order phase transition, cooperativity and is exactly solvable. It also shows an unexpected symmetry between folding and unfolding pathways as suggested by a recent experiment.
\end{abstract}

Proteins function by switching between different well-defined conformations in the native state. In order to reach the native state the protein has to fold. The number of possible conformations is huge, and the existence of folding pathways guiding the proteins between the relevant conformations seems necessary [1]. The existence of pathways has been experimentally established [2, 4 , but the mechanisms behind these are still to be understood. The free energy difference stabilizing the folded state of a protein, is of the order $20 k_{B} T_{\text {room }}$ for a typical domain consisting of about 100 amino acids, meaning that the binding energy per amino acid is only of order of $k_{B} T_{\text {room }}$ 榪. This is a surprisingly small value. The mechanism responsible for stability at very little cost is not known, but has been named "cooperativity" [5].

In this letter we propose to model protein folding by a parametrization of the folding pathway. Cooperativity appears in the theory through assuming that only interlocked degrees of freedom are active in the energy window that separate the folded and unfolded states. This also leads to a first order folding transition.

Furthermore, the model exhibits an interesting dynamical symmetry when changing conformation. One would expect it to follow the folding pathway in opposite direction when it unfolds. However, under certain conditions it moves in the same direction along the folding pathway both when folding and unfolding. We call this the "mirror effect." This feature of the model is in agreement with recent measurements [6] which suggest this mirror symmetry in the conformational change of albumin at low $\mathrm{pH}$.

The amino acids constituting a protein may rotate relative to each other, thus inducing conformational changes in the protein. They furthermore interact. Through changing bond angles, amino acids far apart on the protein may be brought close to each other to form bonds that stabilize a given conformation.

Simple models for protein folding which encompass guiding [7] and cooperativity [8] have been proposed. More complex models based on spin glass hamiltonians [9] need to introduce "folding funnels" in the energy landscape in order to guide the slow dynamics of a frustrated system [10,11]. The existence of a pathway implies that the bond angles change in a certain order, i.e. there is a hierarchy among the bond angles themselves. In order to clarify the significance of such a hierarchy, we introduce a model that simultaneously displays a folding pathway, cooperativity and a first order phase transition, thus reproducing three important characteristics of real proteins. The model may be visualized as in Fig. 11. The variables are effective folding angles $\varphi_{1}$ to $\varphi_{N}$, each associated with matching a given sub part of the protein into its position in the ground state (the template). The key point of the model is that a correct fold at level $i$ gains binding energy when all lower levels are correctly folded. Our angle variables are not to be thought of as the $\phi, \psi$ angles of the polypeptide backbone, but rather as coarse grained variables (i.e. averaged over a small number of monomers). An intuitive picture would be the angle between two $\alpha$ helices connected by a loop. Our model corresponds to the interaction Hamiltonian

$$
H=-a_{1} \varphi_{1}-a_{2} \varphi_{1} \varphi_{2}-a_{3} \varphi_{1} \varphi_{2} \varphi_{3}-\cdots-a_{N} \varphi_{1} \varphi_{2} \cdots \varphi_{N},
$$

where $a_{1}$ to $a_{N}$ are coupling constants.

In order to present explicit calculations we scale all angle variables $\varphi_{i}$ to be between 0 and 1 and set all coefficients $a_{i}$ equal to 1 . Furthermore, we assume for the moment that angle variables only take discrete values, 0 and 1 . The Hamiltonian then represents the number of folded variables $\left(\varphi_{i}=1\right)$ counted from $i=1$ until the first $i=n_{f}+1$ where $\varphi_{i}=0$, and can simply be written

\footnotetext{
*Permanent address: Department of Physics, Norwegian University of Science and Technology, NTNU, N-7034 Trondheim, Norway
} 


$$
H=-n_{f} .
$$

To explore the thermodynamic properties of the model, we calculate its partition function by summing over degenerate states,

$$
Z(T, N)=\sum_{n_{f}=0}^{N-1} 2^{N-1-n_{f}} e^{n_{f} / T}+e^{N / T}=2^{N-1} \frac{\exp (N(1 / T-\ln 2))-1}{\exp (1 / T-\ln 2)-1}+e^{N / T},
$$

where $T$ is temperature measured in units of the coefficients $a_{i}=1$. In the limit $N \rightarrow \infty$, the partition function has a discontinuous derivative for $T=T_{c}=1 / \ln 2$. This means that the system undergoes a first order phase transition at this temperature. At temperatures $T<T_{c}$ the binding energy of the folds dominates and the system freezes into the folded state with $\left\langle n_{f}\right\rangle \approx N$, whereas for $T>T_{c}$ the winning term will be the entropy that is gained from each folded variable, yielding $\left\langle n_{f}\right\rangle \approx 0$, corresponding to an unfolded, molten state. Experimentally, it is known that small proteins typically undergo a first order transition, while for large, multidomain proteins the situation can be more complex [5].

In addition to a phase transition, the model exhibits cooperativity, manifested through the fact that below $T_{c}$, say for $T=1$, the chain is folded even though the binding energy per link is only $k T$, which would lead to a disordered (unfolded) state in the absence of cooperativity. In the present model, at $T<T_{c}$, nearly all states will match the template, with fluctuations only at the bottom of the hierarchy. This matches the findings from real proteins, where cooperativity implies that most degrees of freedom are locked by others.

During a slow "adiabatic" change of temperature through the phase transition, the folding/unfolding follow specific pathways. Under cooling, the first level in the hierarchy must fold first, $\varphi_{1}=0 \rightarrow 1$ in order for the energy to change at all. This is due to the nature of the Hamiltonian where $\varphi_{1}$ is present in all the terms, see (11). After the first, the next level in the hierarchy, $\varphi_{2}$, must fold in order to change the energy further and so on. Under heating, the unfolding will take place from bottom and up, i.e. first $\varphi_{N}=1 \rightarrow 0$ leading finally to $\varphi_{1}=1 \rightarrow 0$. This is due to the fact that the smallest increase of energy is given by the unfolding of the deepest levels in the hierarchy.

Under non-adiabatic changes the unfolding is however different. When $T<T_{c}$, the free energy is

$$
F_{E}(T, N)=-N
$$

as it is dominated by the binding energy. For $T>T_{c}$, the free energy is

$$
F_{S}(T, N)=-T N \ln 2
$$

which results from entropy now dominating. We denote $F_{E}$ the energy branch and $F_{S}$ the entropy branch of the free energy. We may envision these extended beyond the critical temperature $T_{c}$ as shown in Fig. 2. We now perform a quench of the system, i.e., rapidly change its temperature. Starting in equilibrium at a temperature $T<T_{c}$, marked "1" in Fig. 2, and rapidly heat the system to $T>T_{c}$, it moves to "2" in the figure, following the energy branch of the free energy. The system then thermalizes by moving to "3", after which the system ends up on the entropy branch. Starting with the system in equilibrium at a temperature $T>T_{c}$, point "4" in the figure, a rapid quench to a low temperature brings the system to " 5 " along the entropy branch. The system then moves from " 5 " to "6" to reach the energy branch which at this temperature is the lowest free energy.

The question is now how the angle variables react to these two types of quench. When the system is rapidly cooled, the angle variables have to fold in the order $1,2,3, \ldots N$ - otherwise, no change in energy occurs. This is the same order as the system follows during adiabatic cooling. However, when going from low to high temperature the situation is different. When the system moves from "2" to "3" in Fig. 2, the unfolding rate, $\Gamma_{i}$, for each $\varphi_{i}$, is dominated by the size of the phase space of the final state,

$$
\Gamma_{i} \propto e^{i(1 / T-\ln 2)} .
$$

Therefore the rate increases with decreasing $i$, i.e., the rate of change is largest for $\varphi_{1}$, second largest for $\varphi_{2}$ etc. This is the same order as when the system was rapidly cooled and the opposite to the order found in adiabatic melting. We call this reversal of direction "mirror effect." So far as we have regarded the variables $\varphi_{i}$ as binary, as soon as the first angle has unfolded, the others will unfold in no particular order, see Fig. 1. With continuous variables this degeneracy of the model is lifted.

In a recent experiment, an indication of the existence of this "mirror effect" was found [6]. In the experiment, the conformational change induced in albumin by lowering $\mathrm{pH}$ is studied on samples consisting of very few (1-100) 
molecules. The technique is described in detail in [6]; briefly, the molecules are sandwiched between a flat plate and a micron size sphere, and the movement of the sphere relative to the plate, measured to sub nm resolution with an evanescent wave technique, is used to reveal changes in shape (e.g. swelling or contracting) of the molecules in response to a fast $\mathrm{pH}$ change. This corresponds to quenching the proteins into the new state.

Albumin is a large protein $(\sim 600$ aminoacids $)$, consisting of 6 domains. It is believed that the conformational change at low $\mathrm{pH}$ consists essentially in a rearrangement of these domains, so that the shape of the molecule changes (becoming about $60 \%$ longer). However, this is not a complete unfolding: the molecule is still compact, and elements of secondary structure persist. In Fig. 3 we present some measurements from the experiment 6]. As described in [6], the conformational change is seen to proceed through a series of $\sim 5$ steps in time, suggesting a sequential rearrangement of the domains. Thus for this process, a well defined pathway exists.

The experiment further suggests that the reverse conformational change (obtained by restoring neutral $\mathrm{pH}$ conditions) proceeds through the same sequence (not the time reversed sequence) of steps. We observe that the steps occur at similar positions, supporting a mirror imaging of the retracing pathway. In support of this observation, we project in Fig. 1 the trajectory on the distance axis, to show a histogram of the time spend at each distance value, i.e. such that flat regions (waiting times) will be represented by peaks in the histogram. We can then average several histograms. The comparison of the two symmetries, i.e. the "mirror effect" (b) and simple time reversal (c), supports that the proteins under these conditions show the "mirror effect."

In conclusion, we believe to have introduced the first model which simultaneously reproduces three key features of real protein folding, namely a folding pathway, cooperativity and a first order folding transition. In addition the model raises some interesting symmetry discussions (i.e. the "mirror effect").

A.H. thanks Nordita for funding his stay in Copenhagen during which this work was done. Also we like to thank R. Donangelo and K. B. Lauritsen for discussions.

[1] C. Levinthal, J. de Chimie Physique et du Physico-Chimie Biologique 65 (1968) 44.

[2] T. E. Creighton, J. Mol. Biol. 87 (1974) 603.

[3] T. E. Creighton, J. Phys. Chem. 89 (1985) 2452.

[4] A. R. Fersht, FEBS 325 (1993) 5.

[5] P. L. Privalov, Protein folding, ed. by T. E. Creighton, 83 (Freeman, New York, 1992).

[6] G. Zocchi, Proc. Natl. Acad. Sci., in print (1997).

[7] R. Zwanzig, Proc. Natl. Acad. Sci. 92 (1995) 9801.

[8] K. A. Dill, K. M. Fiebig, H. S. Chan, Proc. Natl. Acad. Sci. 90 (1993) 1942.

[9] A. Ansari, J. Berendzen, S. F. Browne, H. Frauenfelder, I. T. Iben, T. B. Sauke, E. Shyamsunder and R. D. Young, Proc. Natl. Acad. Sci., 82 (1985) 5000.

[10] J.D. Bryngelson, J.N. Onuchic, N.D. Socci, P.G. Wolynes Proteins: Struc. Funct. Genet. 21 (1995) 167.

[11] K.A. Dill, S. Bromberg, K. Yue, K.M. Fiebig, D.P. Yee, P.D. Thomas and H.S. Chan, Protein Science 4 (1995) 561.

FIG. 1. The figure describes the refolding of a connected structure, such as a partly unfolded protein, into a well defined template. It illustrates schematically how one by successive foldings of angle variables can match the edges $1,2,3$.. to the template edges $1^{\prime}, 2^{\prime}, 3^{\prime} \ldots$ We suggest to copy the figure and do the folding. One will see that it is not possible to match say edge 2 to template 2', without first matching 1 to 1 '. In general to match $j$ with $j^{\prime}$ demands correct matchings for all $i<j$. This defines a unique pathway from the unfolded to the folded structure, in a way that can be quantified by the Hamiltonian in Eq. (1).

FIG. 2. The free energy per variable. The full line show the equilibrium free energy as a function of temperature. The

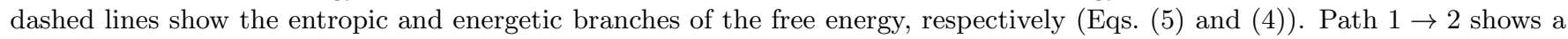
rapid heating of the system. It then unfolds following path $2 \rightarrow 3$. Path $4 \rightarrow 5$ shows a rapid cooling of the system followed by the folding following path $5 \rightarrow 6$. 
FIG. 3. Curves from the experiment [6] showing steps in the dynamics of the forward (left curve) and backward (right curve) conformational change. The ordinate is the height of the sphere above the plate, $h$, in $\mathrm{nm}$, and the abscissa is the time in seconds with arbitrary zero point. For the right curve, only the distance axis has been reversed. The two curves have been placed next to each other for ease of comparison. The dashed lines help identify the steps. The correspondence between the steps in the two curves is the "mirror effect."

FIG. 4. Histograms of the time spent at each distance value, constructed from projecting curves of the type shown in Fig. 3 on the distance axis. The waiting time regions in the original curves then give rise to peaks in the histogram and the distance between two peaks is a step size. We then average several histograms. (a) is an average over histograms corresponding to three unfolding curves. The large peaks at 8 and $11 \mathrm{~nm}$ correspond to the two baselines in Fig. 3. The four peaks in between correspond to the four waiting times shown by the dotted lines on Fig. 3, left curve. (b) is an average over histograms corresponding to three folding curves transformed by reversing the distance axis (i.e. according to the "mirror effect"). Here there are only three peaks corresponding to the three steps in Fig. 3, right curve. As can be seen by comparing histograms (a) and (b) the missing step is either covered by the peak of the base line or simply does not occur in refolding. (c) presents the same histogram as in (b) but with simple time reversal, i.e. no reversal of the diameter axis. We see that (b) leads to better overlap with (a) than (c), indicating that the proteins under these conditions follow the "mirror effect." 


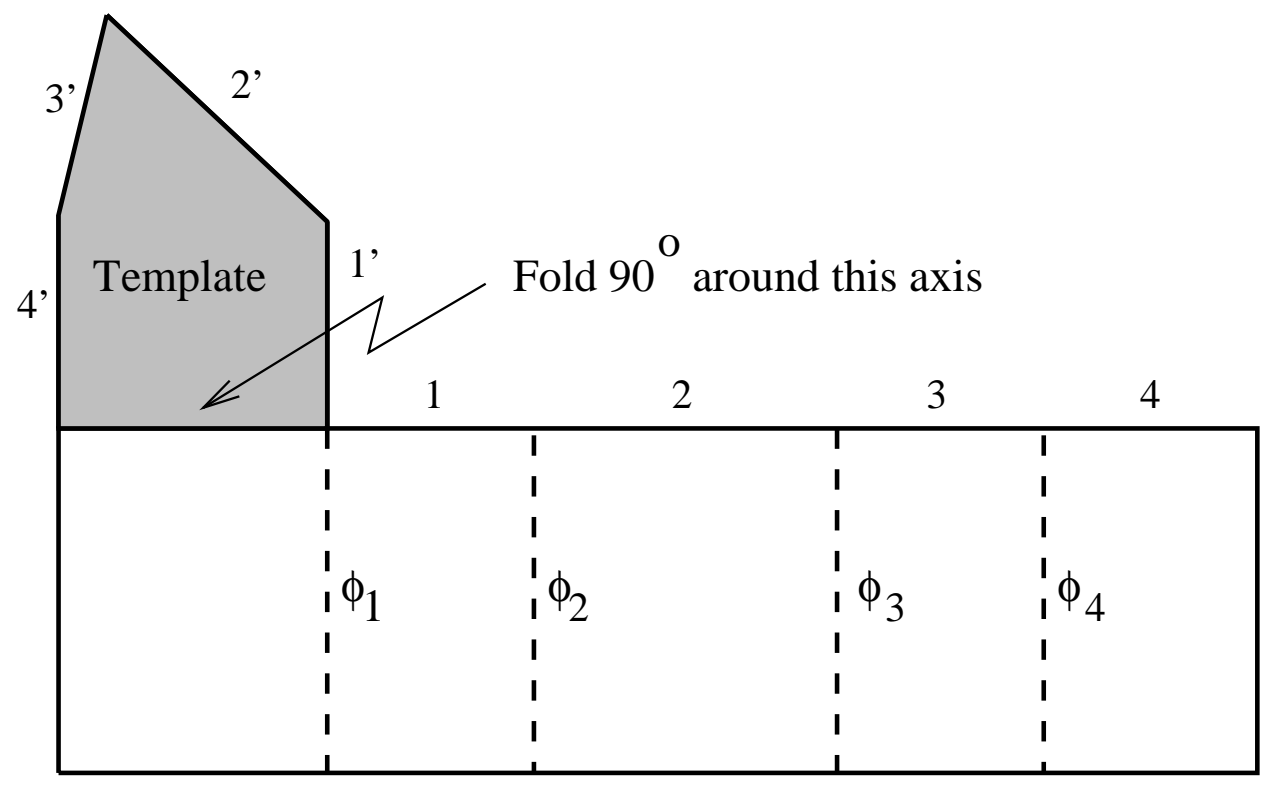

Figure 1

A. Hansen, M.H. Jensen, K. Sneppen and G. Zocchi A hierarchical scheme for cooperativity and folding in proteins 


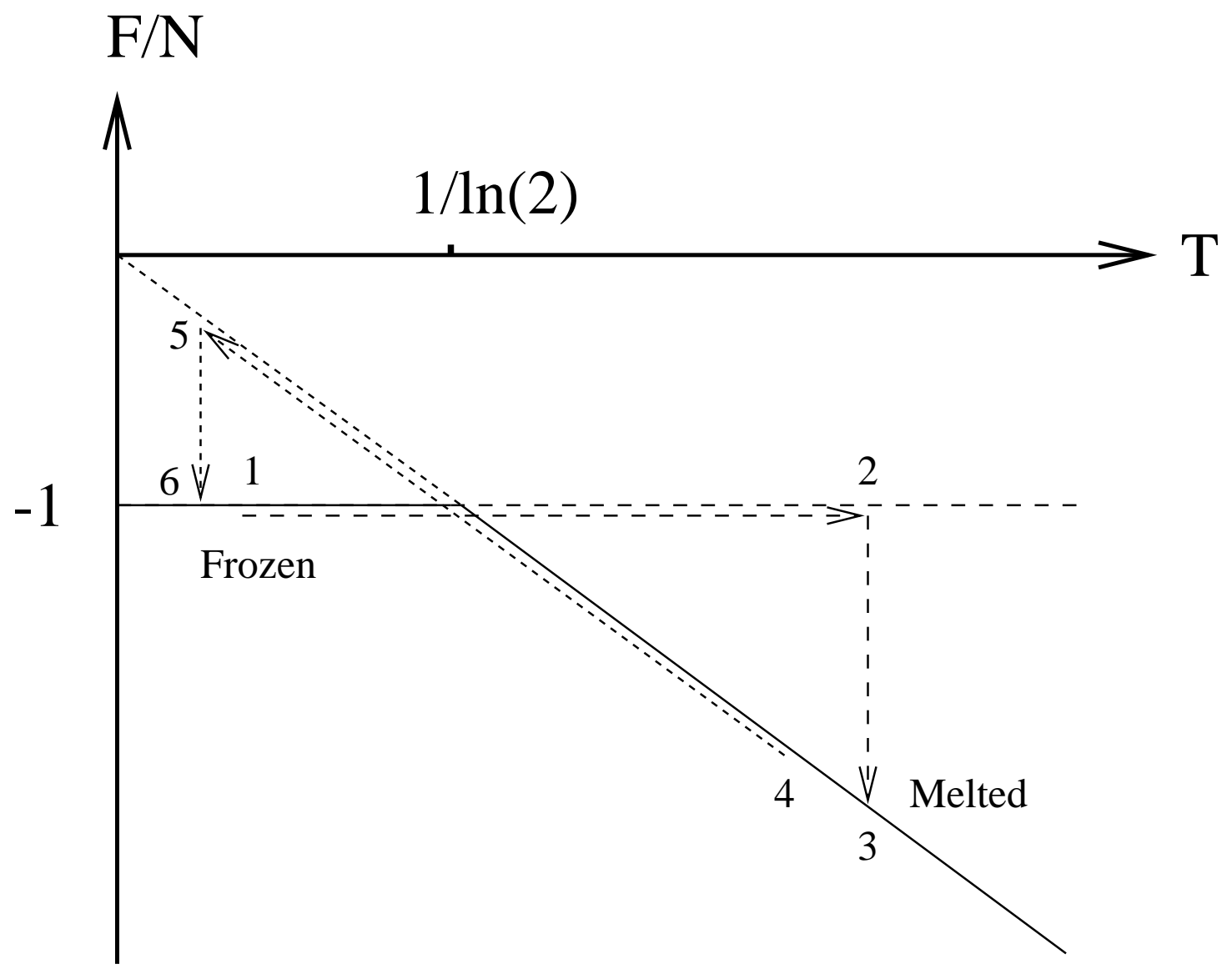

Figure 2

A. Hansen, M.H. Jensen, K. Sneppen and G. Zocchi A hierarchical scheme for cooperativity and folding in proteins 


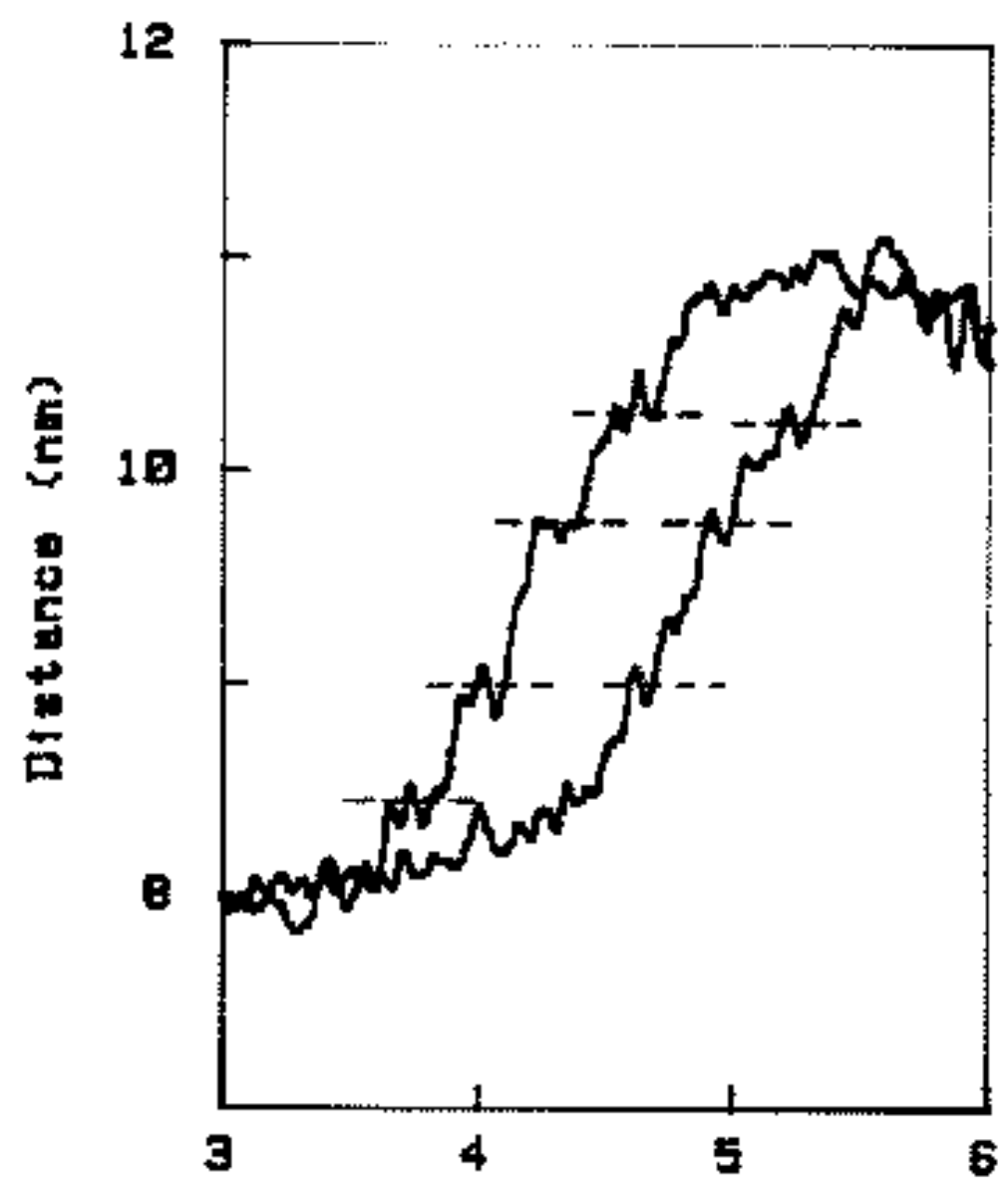

Time $\{x)$

Figure 3

A. Hansen, M.H. Jensen, K. Sneppen and G. Zocchi

A hierarchical scheme for cooperativity and folding in proteins 

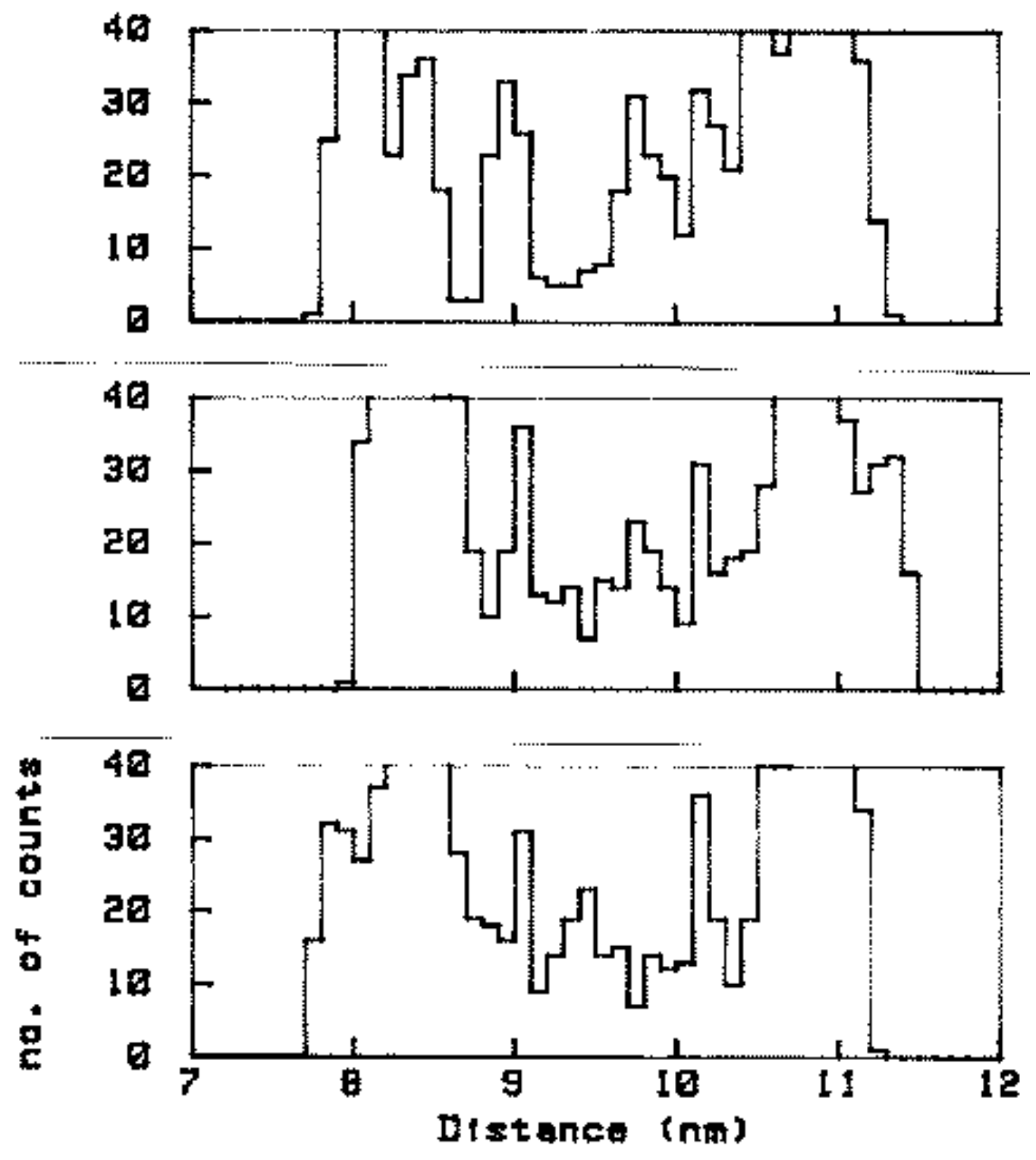

Figure 4

A. Hansen, M.H. Jensen, K. Sneppen and G. Zocchi A hierarchical scheme for cooperativity and folding in proteins 